features, laboratory results of late-onset systemic lupus erythematosus in a tertiary rheumatology center in China.

Methods We retrospectively analyzed 198 patients with lateonset SLE admitted to RenJi Hospital, Shanghai Jiaotong University School of medicine from Jan, 2013-Jan, 2018. A control group of randomly selected 198 SLE patients with disease onset earlier than the age of 50 admitted to the same hospital at the same time period was recruited. Clinical and laboratory data were collected through chart review.

Results Between January 2018 and August 2018, 198 SLE patients fulfilled definition for late-onset SLE. The predominance of women among late-onset SLE (4.7 : 1) was decreased as compared with that observed in early-onset SLE (18.8 : 1). The following clinical manifestations were less common in late-onset SLE when compared to that observed in early-onset group: malar rash $(33.8 \%$ vs $64.1 \%, \mathrm{p}=0.000)$, alopecia $(11.6 \%$ vs $28.2 \%, \mathrm{p}<0.001)$, photosensitivity $(6.06 \%$ vs $13.6 \%, \mathrm{p}=0.011$ ), nephropathy (proteinuria: $24.2 \%$ vs $58.5 \%$; hematuria $2.02 \%$ vs $21.2 \%, \mathrm{p}<0.001$ ), neuropsychiatric symptoms $(1.51 \%$ vs $7.57 \%$., $\mathrm{p}=0.004)$, while a higher incidence of xerostomia existed in late-onset SLE $(9.59 \%$ vs 20.2\% $\mathrm{p}=0.003$ ) (table 1). Complement levels (C3, C4, $\mathrm{CH} 50)$ were significantly higher in late-onset group when compared to those in early-onset group. Immunoglobulin G and A levels were higher in late-onset group than those in early-onset group (table 2). A non-significant decrease of urine protein creatinine ratio was observed in the late-onset group. A significantly increased incidence of positive anti-U1RNP, anti-SSA Ro60, and anti-SSB La was observed among the lateonset SLE patients (table 3).

Conclusions Our study confirmed that in a Chinese population, late-onset SLE patients were different from early-onset SLE patients in terms of clinical manifestations and laboratory results. Funding Source(s): N/A

\section{COMPARING THE PERFORMANCE OF TWO INTERFERON-GAMMA RELEASE ASSAYS IN AUTOIMMUNE SKIN DISEASE PATIENTS: A PROSPECTIVE STUDY}

${ }^{1}$ Rebecca L Krain*, ${ }^{1}$ Rebecca G Gaffney, ${ }^{1}$ Emily R Keyes, ${ }^{1}$ Rui Feng, ${ }^{2}$ Victoria P Werth. ${ }^{1}$ University of Pennsylvania, Department of Dermatology; ${ }^{2}$ Perelman School of Medicine, University of Pennsylvania

\subsection{6/lupus-2019-Ism.170}

Background Autoimmune skin disease patients are standardly screened for tuberculosis via interferon-gamma release assays (IGRAs) prior to starting new immunosuppressive drugs or enrolling in clinical trials. Two commercial IGRAs, T-SPOT. TB and QuantiFERON-Tb Gold (QFT-G), are reported as either determinate (positive or negative) or indeterminate. Both tests utilize similar immunoenzymatic reactions for interferon-gamma detection, but differ in its quantification. Though the QFT-G is more widely used, studies have demonstrated that the T-SPOT.TB has lower rates of indeterminate results in immunosuppressed patients, and thus may prevent a delay in the initiation of necessary therapies or enrollment in clinical trials. The newest generation of QFTG, the QuantiFERON-TB Gold Plus (QFT-Plus), has not been compared to the T-SPOT.TB in this patient population. We aim to investigate the performance of both the T-SPOT.TB and QFT-Plus, as indeterminate results represent a major barrier to receiving appropriate treatment in autoimmune skin disease patients.

Methods This ongoing prospective study included 48 patients seen at the Hospital of the University of Pennsylvania. Venous blood samples were collected from patients and underwent tuberculosis screening with QFT-Plus and T-SPOT.TB IGRAs. The proportions of indeterminate and determinate (positive and/or negative) results among the two tests were compared.

Results In the study population of 48 patients, 29\% had a primary diagnosis of cutaneous lupus $(n=14)$. There were 2 indeterminate results with the QFT-Plus and no indeterminate results with T-SPOT.TB. There was also one positive result that was seen with both the QFT-Plus and T-SPOT.TB. All patients with a primary diagnosis of cutaneous lupus had negative results for both QFT-Plus and T-SPOT.TB. Using a onetailed Fischer test, there was no statistical significance when comparing QFT-Plus and T-SPOT.TB in autoimmune skin disease patients $(p=0.25)$.

Conclusions In this prospective study, the T-SPOT.TB had fewer indeterminate results compared to the QFT-Plus. Though this finding was not statistically significant, it is clinically important as indeterminate results preclude autoimmune skin disease patients from receiving necessary treatment. Compared to previous studies on the older generation of the QFT$G$ test, the QFT-Plus showed improvement in reducing the amount of indeterminate results. Despite this, we suggest using the T-SPOT.TB in tuberculosis screening for autoimmune skin disease patients who have an indeterminant QFT-G or QFTPlus, as this test did not display any indeterminate results. The results of this study are limited by the small sample size. Funding Source(s): This project was supported by Oxford Immunotec, Inc.

\section{CONCORDANCE OF DORIS REMISSION CRITERIA WITH THE TREATING PHYSICIANS (DORIS-)INDEPENDENT REMISSION JUDGMENT IN A SLE-COHORT AT A TERTIARY CENTER}

Johanna Mucke*, Christina Duesing, Nora Kachabia, Gamal Chehab, Matthias Schneider. Policlinic of Rheumatology and Hiller-Research Unit of Rheumatology, Heinrich-HeineUniversity Duesseldorf, Düsseldorf

\subsection{6/lupus-2019-Ism.171}

Background The definition of an accurate target for a treat to target (T2T) approach in SLE has been challenging over the past years. Recently four definitions of remission were presented by the international DORIS task force. Aim of this study was to evaluate the frequency of remission in our outpatient SLE cohort and to assess feasibility and concordance of the remission definitions with the treating physicians opinion regarding the patients state.

Methods In this monocentric cross-sectional study patients with SLE according to the 1997 American College of Rheumatology (ACR) criteria were enrolled and assessed between September 2016 and December 2017. DORIS remission definitions were applied and demographic and laboratory data as 